POLICY IMPLEMENTATION

\section{Divergent perspectives}

Proc. Natl Acad. Sci USA 12, 4911-4916 (2019)

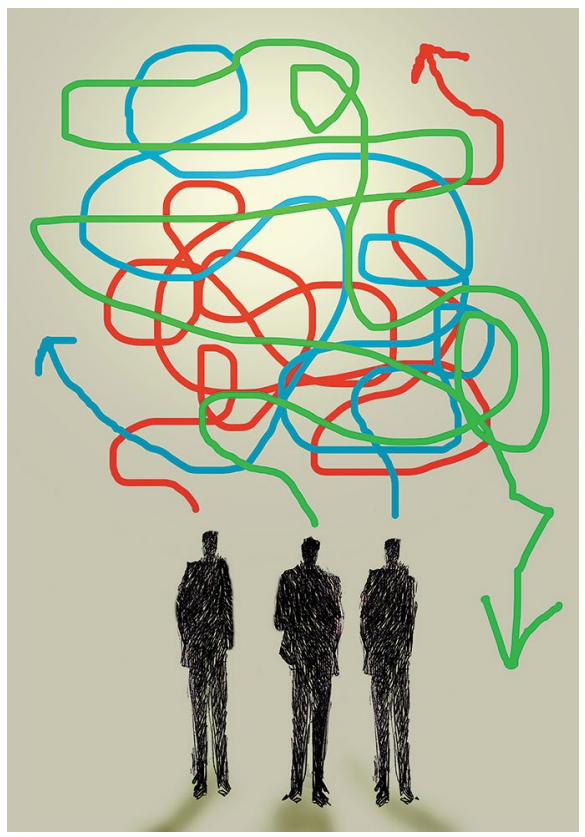

Credit: Ikon Images / Alamy Stock Photo

A major hindrance for successful implementation of sustainability policies, such as the Sustainable Development Goals (SDGs), is that stakeholders disagree over what specific actions to take. Different experiences and knowledge drive each actor to prioritize what needs to be done.
The lack of consensus over priorities (for example, among decision-makers and researchers) can distract our focus from crucial actions. A way of understanding perspectives is to analyse what concepts each actor writes about, such as income, yield or soil quality.

Murray Scown, from Lund University, Sweden, and colleagues, analyse differences and commonalities across perspectives about agriculture and SDGs. They focus on three groups concerned with agricultural systems in Europe: researchers, policymakers and practitioners. To uncover perspectives, they text-analyse research articles, the SDGs, policy documents and sustainability assessment tools. In these documents, they identify 239 social and environmental variables including subsidies, irrigation and soil erosion. Of these variables, just $13 \%$ are shared by all three perspectives. Interestingly, driver variables predominate in research, whereas outcomes are more common in policy documents. These findings highlight the need for intersectoral dialogue to increase agreement over what matters, in order for researchers to produce more useful evidence for policymaking.

\section{Aiora Zabala}

Published online: 9 April 2019

https://doi.org/10.1038/s41893-019-0281-7 